\title{
Acoustic Source Localization in Aircraft Interiors Using Microphone Array Technologies
}

\author{
Bernard J. Sklanka* and Joel R. Tuss ${ }^{\dagger}$ \\ The Boeing Company, Seattle Washington 98124-2207 \\ Ralph D. Buehrle ${ }^{\ddagger}$ and Jacob Klos ${ }^{\S}$ \\ NASA Langley Research Center, Hampton, Virginia 23681-2199 \\ and \\ Earl G. Williams ${ }^{* *}$ and Nicolas Valdivia ${ }^{\dagger \dagger}$ \\ Naval Research Laboratory, Washington, D.C. 20375-5350
}

\begin{abstract}
Using three microphone array configurations at two aircraft body stations on a Boeing 777-300ER flight test, the acoustic radiation characteristics of the sidewall and outboard floor system are investigated by experimental measurement. Analysis of the experimental data is performed using sound intensity calculations for closely spaced microphones, PATCH Inverse Boundary Element Nearfield Acoustic Holography, and Spherical Nearfield Acoustic Holography. Each method is compared assessing strengths and weaknesses, evaluating source identification capability for both broadband and narrowband sources, evaluating sources during transient and steady-state conditions, and quantifying field reconstruction continuity using multiple array positions.
\end{abstract}

\section{Introduction}

L OCALIZING broadband and narrowband sources on aircraft has continually been a challenge for noise engineers. Traditionally sound pressure levels are used to map the aircraft interior. Treatments are then applied in broad strokes over the areas of concern. More recently, the use of matched pair sound intensity measurement techniques have given engineers a slightly higher resolution for determining source characteristics. This technique however has limitations in resolution and dynamic capability.

Over the past decade with the advent of lower cost data acquisition came the development of methods and technologies related to array measurement techniques. Until recently, the use of array measurement techniques inside of aircraft has mainly been limited to strongly correlated or easily referenced sources such as propeller driven aircraft. ${ }^{1}$ The difficulties in using array techniques inside of jet aircraft can be attributed to channel count, source type, the interior acoustic field, and area of coverage.

The utilization of large microphone arrays to identify sources is quite enticing for aircraft manufacturers. Weight reductions and pin-point noise treatments can greatly reduce manufacturing and operation costs and improve airplane performance. To that end, several array methodologies were examined on the Boeing sponsored Quiet Technology Demonstrator 2 (QTD 2) flight test program. These include matched pair intensity measurements, spherical nearfield acoustic holography (SNAH), and conformal "patch" inverse boundary element methods (PATCH IBEM).

The overall process for evaluating the various methodologies starts with a low resolution acoustic 'picture' of the aircraft interior. The low resolution methodologies are used to find general areas of interest. Gradually a higher

\footnotetext{
*Engineer, Aero/Noise/Propulsion Laboratory, bernard.j.sklanka@boeing.com.

${ }^{\dagger}$ Engineer, Aero/Noise/Propulsion Laboratory, joel.r.tuss@ @oeing.com.

${ }^{*}$ Research Engineer, Structural Acoustics Branch, ralph.d.buehrle@ nasa.gov.

$\S^{\S}$ Research Engineer, Structural Acoustics Branch, j.klos@ nasa.gov.

** Senior Scientist for Structural Acoustics, Physical Acoustics Branch, williams@pa.nrl.navy.mil.

${ }^{\dagger \dagger}$ Research Mathematician, Physical Acoustics Branch, valdivia@pa.nrl.navy.mil.
} 
resolution picture is built using successive array techniques. Each acoustic field reconstruction reveals more information about the source characteristics.

\section{Array Development}

In the past, one technical obstacle in the path of the development of an effective near-field acoustical holography system has been the cost and physical size of the measurement microphones available to the engineering community. Because of the large number of transducers needed to populate an efficient array, standard laboratory grade measurement microphones had to be ruled out as too expensive. And when installed in a densely populated array, they could be cumbersome to use, and result in a massive array system that may not be particularly acoustically transparent. With the advent of inexpensive miniature pre-polarized microphones, the Boeing Aero/Noise/Propulsion Laboratory (ANP) was able to successfully develop the various technologies and tools necessary to permit the use of Nearfield Acoustic Holography on test aircraft. This development resulted in prototype planar and conformal holography arrays, and together with groundbreaking work by the Naval Research Laboratory (NRL) and the National Aeronautics and Space Administration (NASA), prototype spherical volumetric acoustic intensity arrays. These technologies were deployed on an airplane in flight in December, 2004 on the NASA 757 out of Hampton, VA. A Boeing-built conformal array and NASA/NRL spherical array along with traditional matched pair intensity probes, various interior microphones, and accelerometers were utilized to validate the usefulness of the array technologies in determining the effectiveness of sidewall noise treatments. The experience gained there helped to further refine the data processing techniques, and produce more efficient and userfriendly next generation conformal and spherical arrays for future airplane testing. The Boeing QTD 2 program offered the opportunity to have access over multiple test flights to a dedicated aircraft test platform from which the conformal and spherical array holography techniques could be further validated and refined.

The QTD 2 program was a Boeing sponsored research effort to focus on advanced technology of noise reduction for future commercial aircraft applications. Conducted in August of 2005, the program was a collaborative effort between Boeing, General Electric, Goodrich, All Nippon Airways of Japan, and NASA. The array technology deployment on the QTD 2 test program was specifically placed on the control side of the aircraft to allow multiple flights of similar configurations to effectively test the multiple techniques.

For this test program, two sets of microphone array instrumentation were used on the interior of the Boeing 777-300ER airplane. Each set of array instrumentation included a 50-microphone 0.4 meter diameter spherical array, a 1.1 meter by 0.5 meter 240-microphone conformal sidewall array, 8 pairs of matched pair intensity probes, and 14 reference transducers (10 accelerometers and 4 microphones). The forward instrumentation set was centered at body station 645 and the aft set was centered at body station 1622 . The aft station array
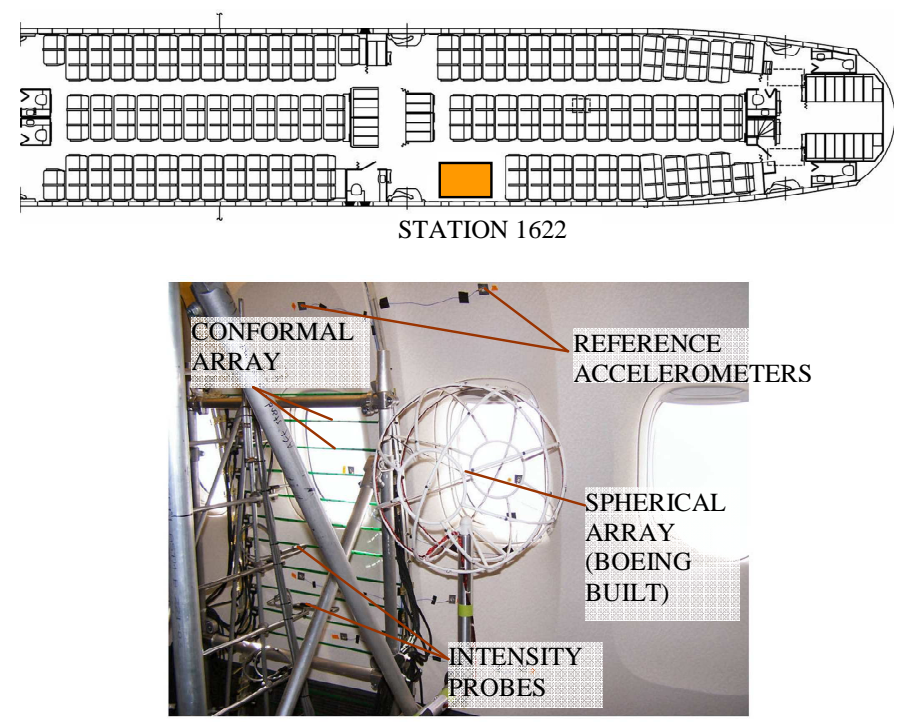

Figure 1. Aft array layout at station 1622. Arrays are located on the control side of the aircraft. configuration is shown in Figure 1.

\section{Conformal Nearfield Acoustical Holography Array Methodology}

The conformal pressure measurement array system consists of an array of variable microphone densities and physical geometries, microphone power supplies with signal conditioning, necessary cabling for attachment to multi-channel digital recording systems, and data processing software. Intended uses include near field measurements of aircraft sidewall sections to utilize back propagation of the measured sound pressure field to derive velocities, pressures and intensities (input power per unit area) on the surface under the array for non-invasively quantifying the vibratory characteristics of aircraft trim panel and insulation treatments, highly detailed sound intensity measurements of various sound sources, and evaluation of noise treatment effectiveness on airplane tests and laboratory tests. By utilizing the forward propagation properties of holography, predictions of the contribution 
of sound-producing patches of interest to the sound pressure levels experienced at arbitrary locations within interior spaces can be made.

\section{A. Theory of Operation}

Conventional conformal Nearfield Acoustical Holography utilizes a discrete number of measurement points closely spaced to a source surface of interest. These methods require that the measurement array completely cover the source in its entirety and that the source is completely on the exterior of the array (sources must not be located on both sides of the array). Ideally this measurement surface would also be closed relative to the source surface. However, practical implementation inside of aircraft does not allow a closed and all encompassing measurement surface. Thus a smaller measurement surface, or "patch" surface, is used instead. An example of this patch approach is shown as the conformal array in Figure 1 above. If the measurement array is denoted as $\Gamma_{0}$ on the surface patch $\Gamma^{p}$ then for a time-harmonic disturbance at a single frequency the sound pressure and normal velocity can be represented for a point $x$ inside the fuselage as ${ }^{2-4}$

$$
\begin{gathered}
p(x)=\int_{\Gamma^{p}} \Phi(x, y) \varphi(y) d S(y) \\
i p \omega v(x)=\int_{\Gamma^{p}} \frac{\partial \Phi(x, y)}{\partial n(x)} \varphi(y) d S(y)+\frac{1}{2} \varphi(x) \\
\Phi(x, y)=\frac{\exp (i k|x-y|)}{4 \pi|x-y|}
\end{gathered}
$$

The boundary surface $\Gamma_{p}$ is decomposed into quadrilateral elements with four nodes. Then the boundary element method with iso-parametric linear functions are selected for interpolating the geometric and acoustical quantities. ${ }^{4}$ Given $\mathrm{M}$ pressure measurements on $\Gamma_{0}$, represented as $p$, recover $\mathrm{N}$ pressure and normal velocity points on $\Gamma_{p}$, represented as $p_{s}$ and $v_{s}$ respectively. When $x$ is a member of $\Gamma_{0}$, Eq. (1) gives the matrix equation

$$
[\mathbf{S}] \varphi=p
$$

where $[\mathbf{S}]$ is a $\mathbf{M} \times \mathrm{N}$ complex matrix and $\varphi$ is the column vector of $\mathrm{N}$ entries that represent values of the density $\varphi$ on $\Gamma$. Similarly, when $x$ is a member of $\Gamma$, Eq. (1), and Eq. (2) produce the matrix equations

$$
\begin{gathered}
\mathrm{p}^{\mathrm{s}}=\left\lfloor\mathbf{S}^{\mathrm{s}}\right\rfloor \varphi \\
\mathrm{v}^{\mathrm{s}}=\left\lfloor\mathbf{K}^{ \pm}\right\rfloor \varphi
\end{gathered}
$$

where $\left[\mathbf{S}^{\mathrm{s}}\right],\left[\mathbf{K}^{ \pm}\right]$are $\mathrm{N} \times \mathrm{N}$ complex matrices. The normal acoustic intensity is derived from $1 / 2 \operatorname{Re}\left(\mathrm{p}^{\mathrm{s}} \cdot \mathrm{v}^{\mathrm{s}}\right)$.

The pressure $p$ contains measurement errors. The numerical solution to the matrix systems in Eq. (4) is ill-posed. The errors in $p$ will be amplified on the solutions $\varphi$ and in most of the cases the recovery of $p_{s}$ and $v_{s}$ will be useless. To avoid the amplification of the measurement errors, special regularization methods are used to find the solution of this linear system. The best known implementation of these methods requires the use of the singular value decomposition (SVD)

$$
\mathbf{S}=[\mathbf{U}][\Sigma][\mathbf{V}]^{H}
$$

where $[\mathbf{U}],[\mathbf{V}]$ are unitary matrices and $[\boldsymbol{\Sigma}]$ is a diagonal matrix containing the singular values $\sigma_{i}$ in order of nondecreasing magnitude. Regularization methods are implemented using the explicit formula

$$
\varphi_{\alpha}=[\mathbf{V}]\left[\mathbf{F}^{\alpha}\right][\Sigma][\mathbf{U}]^{H} .
$$


Here the diagonal matrix $\left[\mathbf{F}^{\alpha}\right]$ contains the filter factors which are used to reduce the effect of the measurement errors in the reconstruction. ${ }^{5}$ The parameter $\alpha \geq 0$ is called the regularization parameter, and should be chosen correctly. There are several types of filter factors used for different ill-posed problems, but in this work we will use the filter factors of Tikhonov with a high-pass filter. ${ }^{6}$ Patch IBEM is implemented using Eq. (8) to solve for $\varphi$ and obtaining $p_{s}$ and $v_{s}$ in Eq. (5) and Eq. (6)

\section{Spherical Nearfield Acoustical Holography Methodology}

The NRL spherical array concept was designed with 50 microphones supported in a spherical shape built around an octahedral solid. Each vertex of each octahedral face lies on the measurement sphere, and those points on the side, face-center, and additional median positions of each octahedral face are projected up to the measurement sphere. The resulting intersections of those projections with the imaginary sphere represent an array of 50 points equally spaced on the spherical contour. The Generation I spherical array (NRL/NASA) and Generation II (Boeing) arrays used on QTD 2 are shown in Figure 2.

The Boeing Generation II spherical array was designed around a different construction technology. Stereo lithography was employed, allowing construction of the spherical microphone

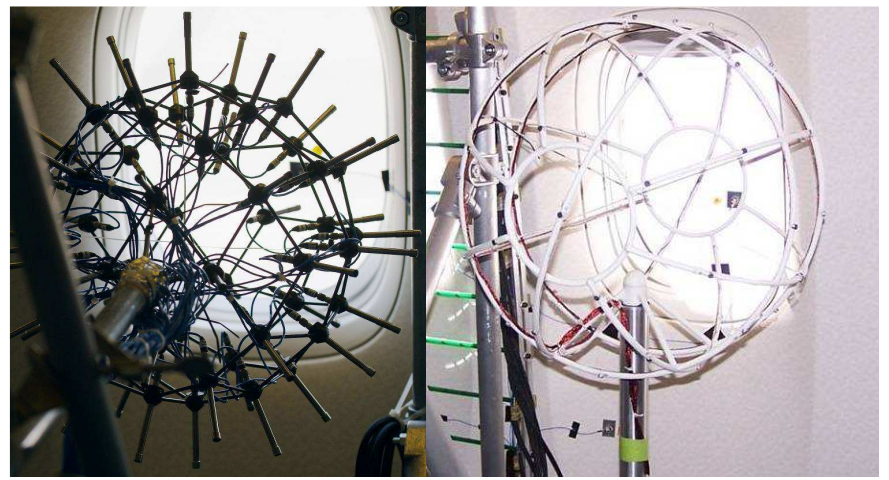

Figure 2. Two spherical array designs. The array on the left was design and build by the Naval Research Laboratory. The array on the right was designed and build by the Boeing ANP Laboratory.

supporting structure to proceed from CATIA drawing directly to finished product, exactly matching the designed dimensions with minimal manual effort and flow time. Because accurate location of the microphones upon the spherical structure is critical to proper operation of the device, small pockets to receive the miniature microphone buttons were built into the design, making accurate positioning of the microphones trivial and foolproof. The total build time required to go from drawing to finished product was less than one man-week. The spherical array is small, light, and portable, and lends itself well to being repositioned during testing. In essence, it can be used as a portable probe to sweep around suspected sources of noise and pinpoint their locations, or get quantitative data on the effectiveness of noise treatments.

\section{A. Theory of Operation}

Spherical Nearfield Acoustical Holography (SNAH) is an efficient algorithm that lends itself well to the fast processing of spherical array pressure measurements into external sound field reconstructions. For QTD 2 with spheres containing 50 microphones, the reconstructed pressures at points external to the array can be described by: ${ }^{7}$

$$
p(r, \theta, \phi)=\sum_{n=0}^{N(\omega, \sigma)} \frac{j_{n}(k r)}{j_{n}(k a)} \sum_{m=-n}^{n} P_{m n}(a) Y_{n}^{m}(\theta, \phi)
$$

where

$$
P_{m n}(a) \equiv \int p\left(a, \theta^{\prime}, \phi^{\prime}\right) Y_{n}^{m}\left(\theta^{\prime}, \phi^{\prime}\right) * d \Omega^{\prime} \approx \sum_{j=1}^{50} w_{j} p\left(a, \theta_{i}, \phi_{i}\right) Y_{n}^{m}\left(\theta_{i}, \phi_{i}\right)^{*}
$$

Here, the pressures at the 50 microphone locations on the measurement sphere (radius $a=0.2 \mathrm{~m}$ ) are integrated using the Lebedev quadrature algorithm to determine the unknown Fourier coefficients $P_{m n}$. The algorithm employs weighting functions, measured pressures $\mathrm{p}$, and spherical harmonics to approximate $P_{m n}$. The integration is exact if $\mathrm{N} \leq 5$ (50-microphone sphere). Thus, as long as the reconstruction radius is less than approximately twice the measurement sphere radius, and intersects no physical boundaries, the reconstructed pressures can be quickly and accurately determined. 


\section{Matched Pair Intensity Microphone Methodology}

Single axis intensity measurements using the matched pair intensity approach have been used extensively to characterize noise sources inside aircraft. Resulting data are typically processed in $1 / 3$ octave bands with finite difference and sums to calculate the velocities and pressures. The intensity equation for this measurement technique is ${ }^{8}$

$$
\mathbf{I}_{\text {probe }}=p \times \mathbf{v}_{\text {probe }}=-\frac{p_{2}+p_{1}}{2 \rho_{0} \Delta r} \int_{0}^{t}\left(p_{2}-p_{1}\right) \mathrm{dt}
$$

where $p_{2}$ and $p_{1}$ are the measured pressures of the matched pair intensity probe, $\Delta r$ is the microphone spacing, and $\rho_{0}$ is the density in air. For airplane testing, the two most limiting factors for this technique are frequency limitations arising from the microphone spacing and the dynamic range of the measurement (the highest level of active intensity relative to the lowest level measurable) resulting from phase error and random error of the measurement system.

Multiple fixed pair intensity probes were used on the QTD 2 test. The intensity pairs are shown in Figure 1. Seven pairs were positioned directly behind the conformal array allowing a low resolution and high resolution measurement to be compared.

\section{Results and Discussion}

The overall process for identifying sources is normally a low to high resolution approach. The lowest resolution method is SPL mappings of the cabin using single point microphones. Once broad regions of concerns are identified, a fixed or scanned intensity system, consisting of a closely spaced phased matched microphone pair, is used to identify the highest source locations. Multiple microphone array techniques are employed to give a detailed look at the areas of most concern (Figure 3). Each of these array methodologies brings to the table strengths and weakness, and each lends itself to specific testing types or scenarios.

Form factor continues to be an issue with the conformal array. Significant reduction in size and weight compared to our early prototypes has been achieved, and the use of miniature microphones cartridges have resulted in acoustically transparent, lighter, simpler transducer arrays. Incorporating narrow thin circuit boards as microphone support structures has allowed the elimination of separate plastic or metal supporting structures and the heavy, unwieldy cabling to route power and signals from the microphones. Nevertheless, the array
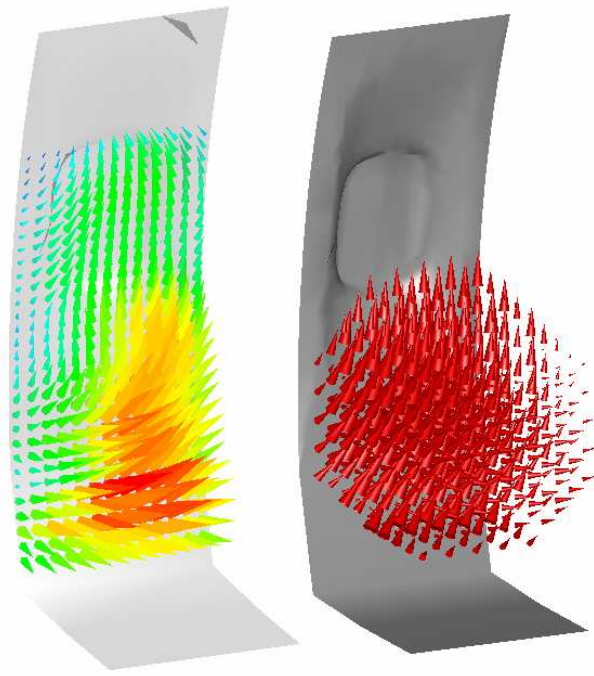

a)

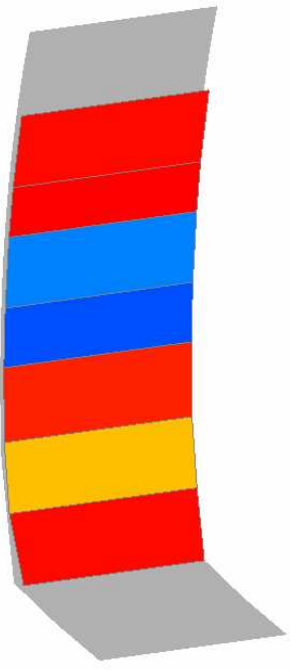

c)

Figure 3. Three reconstructions of aircraft intensity field during a flight test condition: a) PATCH IBEM NAH, b) SNAH, c) fixed match pair intensity (shades of red indicating intensity into the cabin, blue indicating negative or negligible intensity). The succession of resolutions help identify sources of interest. necessitates the removal of seats for airplane installation. Roving the array to several locations within an airplane in flight has been proven, but is not a simple or efficient process. Because of the large number of measurement points on a conformal array, typically 128 or multiples thereof, the size and weight of the coaxial cabling leading to the recording system can be substantial.

As the number of transducers is typically large, the processing power required for the digitization and domain transformation of the raw samples can be substantial. The conformal approach requires external references to phase correlate the data. These can be accelerometers on the measured surface, force cells on vibration source instrumentation, or various field microphones, but additional effort is necessary to acquire and manage these extra signals and process the results. 
Geometry data for the surface under test and the microphone array must be collected and managed, and all transducer measurements and ancillary data sets must be fed into computationally intensive inverse boundary element processing algorithms. Thus, the processing of measured data into reconstruction holograms is fairly limited to a non-real-time post processing mode of operation. Also because of the process intensive algorithms the PATCH IBEM NAH is not the most suited for transient analysis.

All current Boeing conformal arrays are single layer, i.e., not representing a closed analytical surface. Consequently, they suffer from an inability to distinguish between a noise source on the surface under test (in front of the array), and a source in back of the array structure elsewhere in the fuselage interior, e.g., on the opposite fuselage sidewall. A quantifiable result can only be closely approximated, not quantitatively exact with a single layer conformal array. Typically this has not been a major issue, as the close proximity of the microphones to the measured surface of interest usually results in significantly higher sound pressure levels from those sources, thus rendering the effects of other interior sources less significant. The problem exists nonetheless, and may be addressed in future array designs.

The greatest strength to the conformal array methodology, and one verified over multiple tests, is its high resolving capability. With its larger number of array microphones all sampling within the evanescent field over a large range of frequencies, a high resolution intensity map of the extant sound field is routine, and is a valuable tool in discerning accurate location of sources on a measured surface. As well, the conformal methodology because of its ability to support high transducer density in multiple dimensions permits frequency ranges in excess of those possible with the spherical method.

While the conformal array techniques have their merits the necessity of having an area of interest to study is limiting when starting from an unknown source mapping. The Spherical Nearfield Acoustical Holography system provides a necessary link between the lower resolution intensity wands to the high resolution conformal techniques.

The ability to rove the sphere allows the ability of building volumetric radiation characteristics through multiple array positions. Figure 4 shows the multiple sphere positions of the aft array system used during a long steady-state cruise condition on the QTD 2 flight test. Because of the need for references for the processing, this roved technique is not well suited for transient analysis but showed coherent field characteristics for the steady-state condition.

A single position of the SNAH array can be used to evaluate the general field characteristics by doing a pseudotransient analysis. This process is useful in analyzing transitory fields such as those incurred during take off. Figure 5 shows a single frame of an animation taken during a transient take-off condition. A wide frequency band was chosen to capture the change of the engine tone during the condition.

As well, SNAH can be used to identify energy flow within the reconstruction area. This energy flow is the true three-dimensional flow field within the reconstruction aperture, and is directly related to the microphone density and sphere radius. One disadvantage of the SNAH method is poor resolution relative to the conformal method. Depending upon frequency, many of the microphones in the array are not in the near field, and the evanescent components to the field are lost, resulting in limited source resolution ability. The overall accuracy of the projection depends upon two factors. At lower frequencies, the signal-to-noise ratio determines the absolute accuracy of the reconstruction. For higher frequencies, the microphone spacing establishes the limit. Increasing the number of microphones will reduce that error.

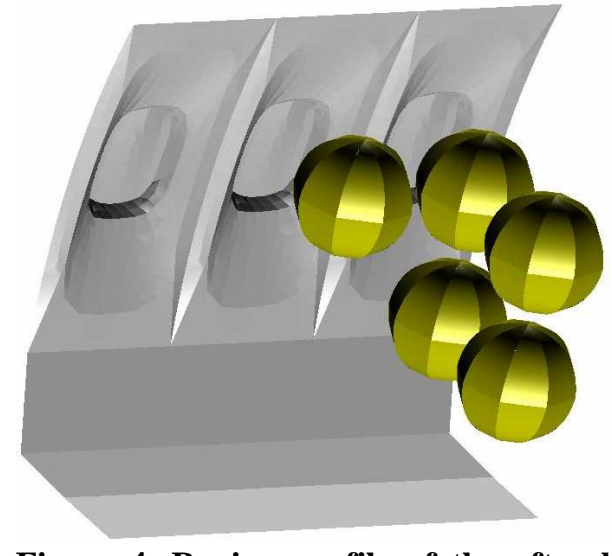

Figure 4. Roving profile of the aft spherical microphone array.
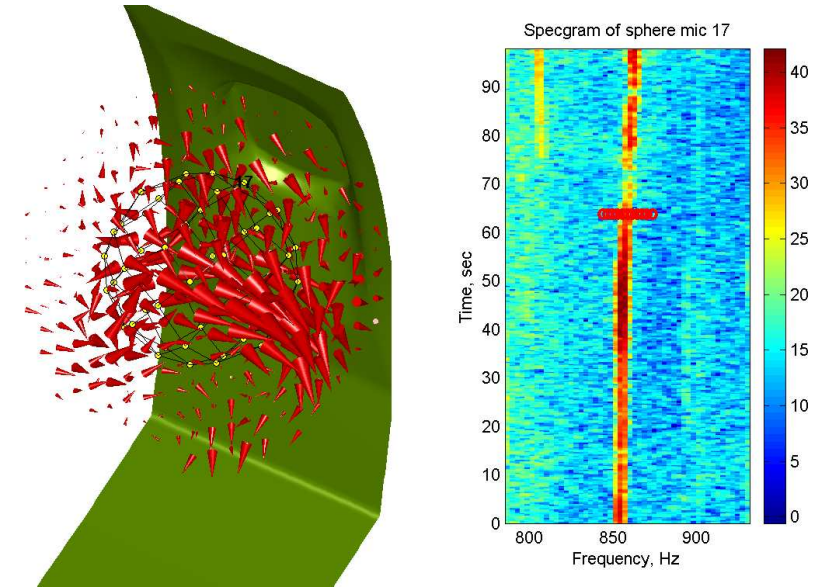

Figure 5. Snapshot of transient animation using forward sphere and SNAH processing. 
The spherical system can still be limiting in terms of system size and operation. This is especially true on flights where limited time and access makes a truly portable system desirable. A severe step in resolution (both frequency and spatial) comes with the matched pair intensity system. This intensity "wand" is used extensively in the acoustic industry, and because of the accepted practice is often times the tool of choice for airplane testing. The ease of use and the portability of the system make it ideal to rove through the aircraft. International standards, when used, can help limit measurement errors. Many systems process and display the data in real time, making this tool quite useful when a fast answer is needed.

However, there are serious limitations to data acquired with the matched pair intensity system. For the traditional $1 / 3$ octave intensity measurement using standards, spatial resolution and dynamic range of the intensity measurement limit the source identification on aircraft to only the highest levels over broad areas. Additional configurations can be used, such as fixed intensity positions and correlated intensity to improve resolution and dynamic range. These additional options however are time consuming to setup and each additional intensity pair is several thousand dollars making large area coverage cost prohibitive. Also, narrowband sources are poorly resolved using this approach which make the higher resolution methods necessary.

\section{Conclusion}

Overall the QTD 2 flight test was a success in validating and demonstrating the various array technologies developed by Boeing, NASA, and the Naval Research Laboratory. The matched pair intensity approach will continue to be the dominant method used because of the ease of operation and portability of the system. However the Spherical Nearfield Acoustical Holography system will start to approach the ease and portability of the intensity system as technology develops. Thus the SNAH approach holds the most promise for fast, reliable, and high resolution data. Finally, the conformal array methodologies will continue to be the best techniques to achieve the highest resolution data. But the cost, time, and effort to deploy these systems and process the data will keep this technology as the final measurement step when high resolution data is needed to resolve a localized noise source on the aircraft.

\section{References}

${ }^{1}$ Williams, E. G., Houston, B. H., Herdic, P. C., Raveendra S. T., and Gardner, B., "Interior near-field acoustical holography in flight", Journal of the Acoustical Society of America, Vol. 108, No. 4, 2000, pp. 1451-1463.

${ }^{2}$ Colton, D. and Kress, R., Integral Equation Methods in Scattering Theory, Wiley-Interscience Publication, New York, NY, 1983.

${ }^{3}$ DeLillo, T. K., Isakov, V., Valdivia, N., and Wang, L., "The detection of surface vibrations from interior acoustical pressure," Inverse Problems, Vol. 19, No. 3, 2003, pp. 507-524.

${ }^{4}$ Valdivia, N. and Williams, E. G., "Implicit methods of solution to integral formulations in boundary element methods based near-field acoustic holography," Journal of the Acoustical Society of America, Vol. 116, No. 3, 2004, pp. $1559-1572$.

${ }^{5}$ Hansen, P. C., Rank-Deficient and Discrete Ill-Posed Problems, Siam, Philadelphia, PA, 1998.

${ }^{6}$ Williams, E. G., "Regularization methods for near-field acoustical holography," Journal of the Acoustical Society of America, Vol. 110, 2001, pp. 1976-1988.

${ }^{7}$ Williams, E. G., Fourier Acoustics: Sound Radiation and Nearfield Acoustical Holography, Academic Press, London, UK, 1999.

${ }^{8}$ Fahy, F. J., Sound Intensity, Second Edition, Spon Press, New York, 1995 